\title{
The Effect of Discontinuous Methanol Addition on the Growth of a Carbon-limited Culture of Pseudomonas
}

\author{
By J. D. BROOKS* AND J. L. MEERS \\ ICI Agricultural Division, Research and Development Department, \\ Billingham, Teesside
}

(Received 2 March 1973)

\begin{abstract}
SUMMARY
As the interval between methanol additions to methanol-limited cultures of Pseudomonas methylotropha was increased beyond $20 \mathrm{~s}$ the yield of bacterial dry wt/g of methanol fell significantly. Discontinuous methanol additions also caused cycling in the values of a number of parameters, including $\mathrm{pH}$, dissolved oxygen tension, $\mathrm{CO}_{2}$ production and amino acid pool concentration. It is suggested that after each addition of methanol a burst of growth occurred, followed by a period of starvation. These observations are discussed in the light of continuous culture practice and theory.
\end{abstract}

\section{INTRODUCTION}

Oscillations in continuous culture populations of micro-organisms have been reported by many workers and can be of two basic types. In one case biotic factors bring about fluctuations in parameters such as population density or oxygen tension without any externally induced change in the growth environment. Under these circumstances, oscillations about mean (as distinct from equilibrium) states have been observed. Classical examples of such oscillating systems have been observed when some mixed cultures of organisms are allowed to grow in continuous flow systems (see Meers, 1973). Harrison \& Pirt (1967) observed sustained oscillations in dissolved oxygen tension in axenic chemostat cultures of Aerobacter aerogenes with a periodicity of about 5 h. Degn \& Harrison (I969) explained such oscillations in terms of variations in respiration rate. In this model the conditions during the experiments were considered to be analogous to those bringing about oscillations in electrochemical systems.

In the second case, oscillations occur in continuous cultures following disturbances in the feed rate of various essential nutrients. Mateles, Ryu \& Yasada (1965) conducted 'shift up' experiments by suddenly increasing the dilution rate of a culture. They found that a large increase in dilution rate resulted in a transient increase in supernatant ammonia (the nominal growth-limiting substrate) concentration. The growth rate did not immediately reach the new value, but increased rapidly by $0.2 \mathrm{~h}^{-1}$, and then took several hours to reach the new steady-state value. If the shift up in dilution rate was less than $0.2 \mathrm{~h}^{-1}$ then the new steadystate growth rate was reached immediately. These results were taken to indicate that protein and RNA biosynthesis were not operating at their maximum capacities under the steady conditions at the time immediately preceeding 'shift up'. Nagai, Nishizawa, Endo \& Aiba (1968), Gilley \& Bungay (1967) and Regan, Roper \& Moss (I97I) have also conducted 'shift up' experiments and observed damped oscillations of specific growth rate. The

\footnotetext{
* Present address: Department of Chemical Engineering, University of Sydney, New South Wales, 2006, Australia.
} 
classical Monod model for bacterial growth does not predict oscillations in growth rate of bacterial concentration either following stepwise increases in dilution rate or following the sudden injection of a quantity of the growth-limiting substrate (Koga \& Humphrey, I967).

Periodic feeding of growth-limiting substrates causes the synchronization of division of microbial populations (Dawson, 1965; Goodwin, 1969). Dawson (197I) has developed apparatus for achieving continuous phased growth in which nutrient additions occur only once during each individual growth cycle. When micro-organisms were grown under such conditions, various parameters (such as dissolved nutrient levels) oscillate with a frequency corresponding to the interval between nutrient additions. Hanche (I969) has suggested a theoretical basis for the entrainment of populations growing under conditions of intermittent nutrient supply. He argues that, since individual growth rates depend on physiological age, then oscillation of a limiting concentration of essential neutralite over the individual growth cycle acts so as to entrain the growth of the organisms.

Meers (I97I) conducted 'shift up' and 'shift down' experiments with mixed cultures and obtained complementary oscillations in the microbe densities of the species in the mixtures. To account for this type of oscillation he postulated that the two species in the mixture had saturation curves that crossed at low dilution rates.

The present investigation was prompted by the observation that regular oscillations in $\mathrm{pH}$ and dissolved oxygen tension occurred in carbon-limited cultures of Pseudomonas methylotropha growing continuously at low dilution rates. The frequency of these oscillations was the same as the frequency of addition of the methanol carbon source. From this observation it seemed possible that the organisms were growing discontinuously rather than in the regular fashion assumed in most theoretical treatments of bacterial growth in chemostats. However, the situation was not one of synchronized growth as described by, for example, Dawson (1971); this study concerns the kinetics of discontinuous population (rather than individual) growth in an environment subject to regular short-term changes. A preliminary report of this work has been published (Meers \& Brooks, 1973).

\section{METHODS}

Organism. The strain of Pseudomonas methylotropha, isolated from soil and kindly provided by Dr T. R. Owen, was maintained on methanol agar slopes at $37^{\circ} \mathrm{C}$.

Media. The carbon (methanol)-limited continuous culture media were described by MacLennan, Ousby, Vasey \& Cotton (I97I).

Continuous culture equipment. The basic design of the equipment was described by MacLennan et al. (197I). However, by adjusting a series of timers, pumps and clips, the mode of addition of methanol and nutrient salts solutions could be altered in four ways. (i) The methanol was continuously pumped into the nutrient salts feed line so as to give a continuous addition of all nutrients into the growth vessel. (ii) The methanol and the nutrient salts solution were pumped continuously into a catch-pot. The contents of the catch-pot were then pumped rapidly into the fermenter at pre-set intervals. This meant that both methanol and the salts solution were added discontinuously. (iii) The methanol only was added to the catch-pot, and pumped discontinuously into the fermenter, while the nutrient salt solution was added continuously to the fermenter. (iv) Methanol was pumped continuously to the culture while the nutrient salts were added intermittently via the catchpot as described above. Since the bulk of the liquid was added to the fermenter with the nutrient salts solution, an appreciable time elapsed between methanol additions because a drop of methanol had to form before it entered the culture. The interval between methanol additions was $5 \mathrm{~s}$. 


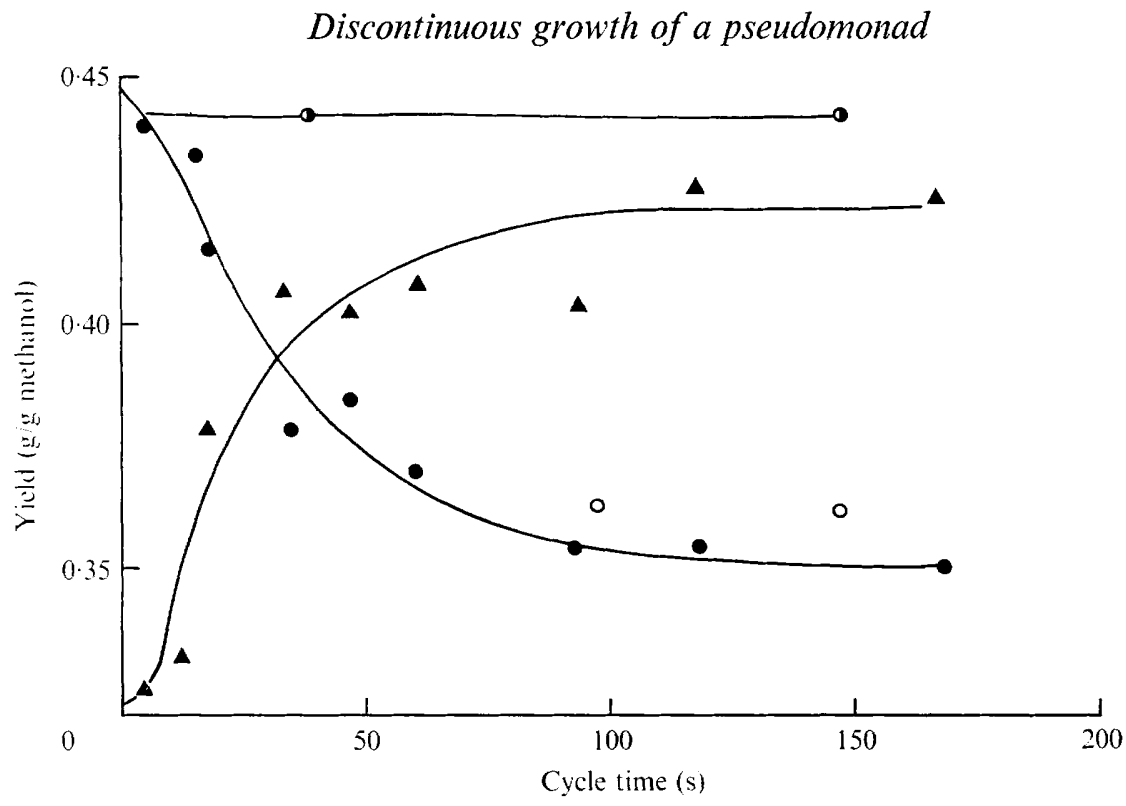

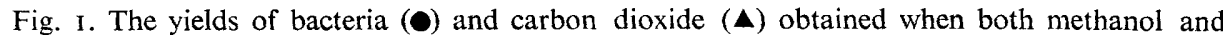
nutrient salts were added intermittently to continuously growing Pseudomonas methylotropha. When only the nutrient salts were added discontinuously the yield of organisms was not altered significantly (O). When only the methanol was added discontinuously while the nutrient salts solution was added continuously the organism yield fell significantly $(\mathrm{O})$.

The dilution rate was $0.23 \mathrm{~h}^{-1}$; the temperature was $37^{\circ} \mathrm{C}$ and the $\mathrm{pH}$ was $6 \cdot 8$.

Analytical procedures. Bacterial dry weights were determined by centrifuging $5 \mathrm{ml}$ portions of culture for $45 \mathrm{~min}$ at $\mathrm{I} 8000 \mathrm{~g}$ at $4{ }^{\circ} \mathrm{C}$, washing with distilled water, recentrifuging and drying for $\mathrm{I} 6 \mathrm{~h}$ at $105{ }^{\circ} \mathrm{C}$. Duplicate estimations agreed to within $\mathrm{I} \%$.

The concentration of methanol in the sterilized methanol feed was determined by specificgravity measurements. The accuracy was $\pm 0 . \mathrm{I} \%$. The residual concentration of methanol in the culture supernatant fluids was determined by vapour phase chromatography. Methanol assimilation, subsequent to sampling, was stopped by the addition of $0 \cdot \mathrm{I} \mathrm{ml}$ of a saturated aqueous solution of $\mathrm{HgCl}_{2}$ to the $10 \mathrm{ml}$ sample.

Dissolved $\mathrm{CO}_{2}$ was measured with an EIL 8002-2 electrode (EIL Ltd, Richmond, Surrey).

Pool amino acids were estimated by the method of Tempest, Meers \& Brown (I97I). A simple, rapid sampling technique was devised so that six serial samples could be removed from the fermenter during one complete cycle of methanol injection. Each $5 \mathrm{ml}$ sample was withdrawn directly into a syringe and transferred as quickly as possible into $0.75 \mathrm{ml}$ of chilled $2 \mathrm{M}-\mathrm{HClO}_{4}$. This operation took less than $5 \mathrm{~s}$. The concentration of amino acids in the culture supernatant fluid was negligible when compared with the pool amino acid concentration.

\section{RESULTS}

When methanol and medium were both added to the fermenter continuously (mode I) the average steady state yields were 0.44 equiv. dry wt/g methanol assimilated, and $0.48 \mathrm{~g}$ $\mathrm{CO}_{2} / \mathrm{g}$ methanol respectively. But as the interval between the additions of methanol and nutrient salts was extended (mode II) the methanol yield figure fell until, when methanol was added every $100 \mathrm{~s}$, a yield of about $0.35 \mathrm{~g}$ organisms/g was reached (Fig. I). Intervals longer than this did not give even lower yields. The carbon dioxide yield increased con- 


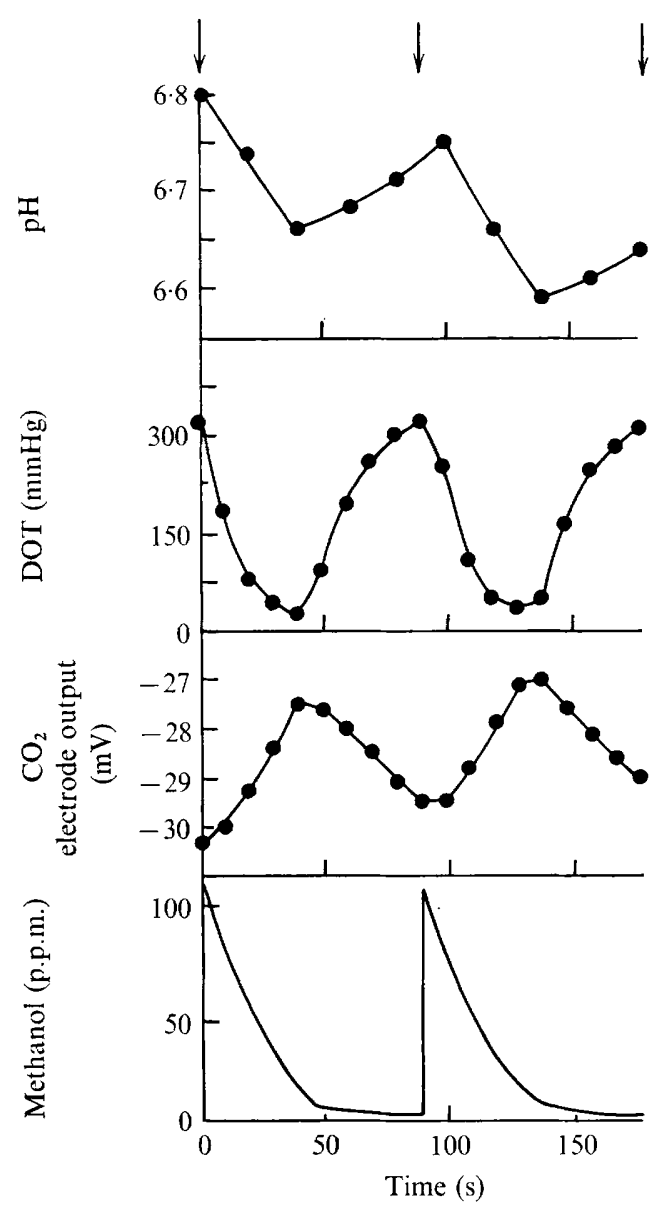

Fig. 2. Variations with time in $\mathrm{pH}$, dissolved oxygen tension (DOT), carbon dioxide production and estimated extracellular methanol concentration. Methanol was added to the fermenter culture at the times indicated by arrows.

comitantly as the dry yield decreased (Fig. I). This first series of experiments were not designed to show which nutrient(s) caused the drop in yield when the medium was added discontinuously. To see whether the growth-limiting substrate (methanol) was responsible, two further series of experiments were done in which either methanol or the other nutrients were added to the culture discontinuously. It was the method of methanol addition which caused the variations in yield (Fig. I).

Following each methanol addition a distinct cycle of changes took place (Fig. 2). During the first part of the cycle the dissolved carbon dioxide level increased, while the $\mathrm{pH}$ and the dissolved oxygen tension (DOT) decreased. After a distinct interval (which was constant when methanol was added with a given frequency) all of these trends were reversed until the next methanol addition. The interval, between methanol addition and the time when the above-mentioned trends were reversed, was considered to be the unrestrained growth period and the second part of each cycle termed the starvation period. When the overall growth rate was maintained at $0.23 \mathrm{~h}^{-1}$ and the periodicity of the methanol additions was 


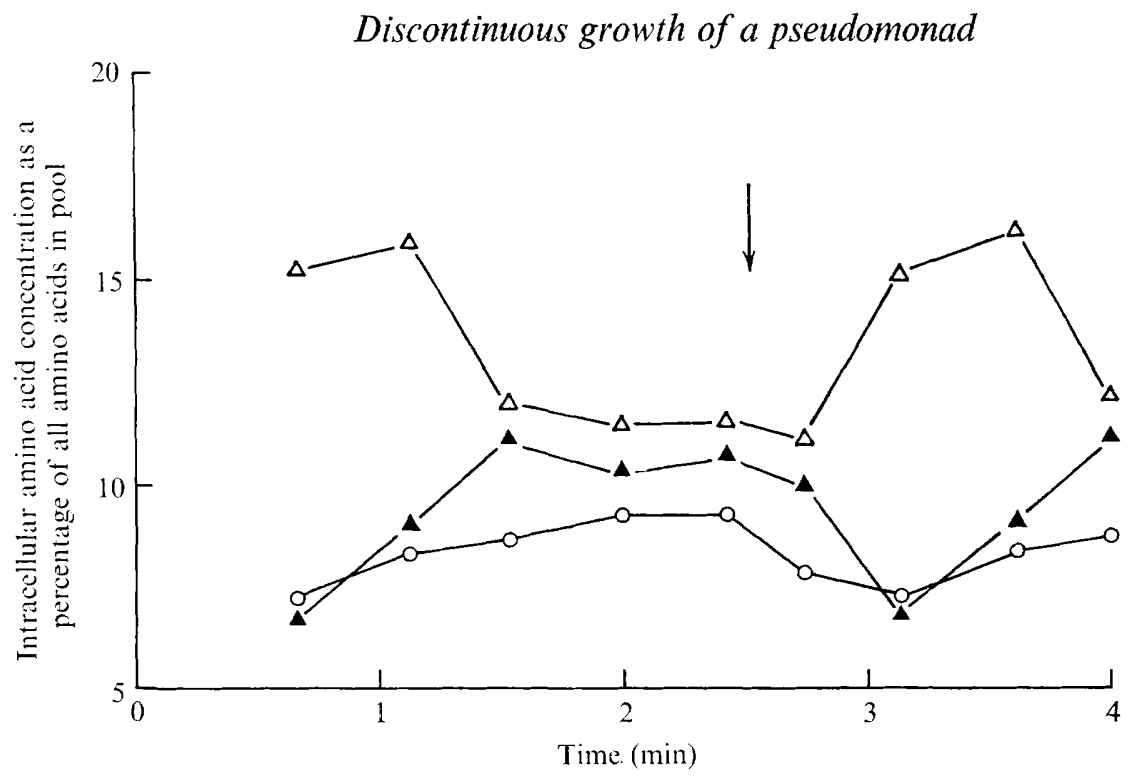

Fig. 3. Variations in pool glutamate $(\Delta)$, glutamine $(\boldsymbol{\Delta})$ and aspartate $(O)$ concentration with time. Methanol was being added to the culture at $138 \mathrm{~s}$ intervals and the arrow marks the point of one addition.

varied (cf. Fig. I) the ratio between the unrestrained growth period and the time for one complete cycle was a constant $(0 \cdot 45)$.

The level of pool amino acids was found to give useful information with regard to the physiological state of populations of micro-organisms (Brown, MacDonald-Brown \& Meers, 1973). When the concentration of pool amino acids was measured in the discontinuously growing pseudomonads, cyclic fluctuations in the glutamine and glutamate levels were observed (Fig. 3). The levels of the other amino acids did not vary significantly. This species has been shown to incorporate ammonia into glutamate, under these growth conditions, via glutamine using the enzymes glutamine synthetase and a constitutive NADlinked glutamate synthase (C. M. Brown \& J. L. Meers, unpublished observation), using the cyclic route described by Tempest, Meers \& Brown (1970). Hence, all of the amino nitrogen that became incorporated into protein had to pass through the intermediates glutamine and glutamate, and therefore it might be anticipated that the level of these amino acids would be sensitive to irregularities in the growth of this species.

\section{DISCUSSION}

When methanol was added at intervals to methanol-limited cultures of the pseudomonad, growth was not continuous. Growth occurred only after each methanol addition, during which time the organisms assimilated oxygen and produced carbon dioxide. This result is to be expected if growth rate is related to substrate concentration as predicted by Monod (I942) but the drop in yield with increase in the periods between methanol additions was unexpected. Two explanations for this drop can be proposed. Firstly, this species is known to give higher yields when grown under carbon-limited conditions than when grown under conditions of carbon excess (J. D. Watts \& J. L. Meers, unpublished observations). When grown under the cyclic conditions described above, the organisms were growing in the presence of excess methanol for much of the time. It is also interesting that the yield of 
$35 \%$ that was obtained when methanol was added at intervals of over $100 \mathrm{~s}$ was the same as the yield obtained by Watts \& Meers (unpublished observations) for this species when grown under nitrogen-limited conditions in the presence of excess methanol. According to this explanation, the main reason for the low yields described above was that the organisms were 'wasting' excess methanol by converting it into carbon dioxide, methanol being a potential growth inhibitor.

Secondly, it is also possible that when the organisms were growing intermittently they were constantly trying to readjust their enzyme and key intermediate levels as they were first carbon limited and then unrestricted in their growth. Under such conditions enzymes and intermediates might be synthesized and degraded in a wasteful, irregular way that would lead to lowered yields. In favour of such a conclusion was the observation that the pool glutamine and glutamate levels fluctuated in a cyclic manner, and both of these intermediates have been reported to control certain enzyme systems (Brown et al. 1973). Glutamate has been shown to be produced in greatest quantities by rapidly growing organisms (Tempest et al. 1971), and glutamate was formed in the discontinuously growing organisms following each methanol addition. When the methanol had been assimilated, presumably the level of citric acid cycle intermediates became lower, and that was why the rate of glutamate production decreased. Indeed some glutamate may well have been converted back into $\alpha$-oxoglutarate as an energy source during the starvation period. Glutamine would have been expected to accumulate during the starvation period because of the lack of $\alpha$-oxoglutarate as an acceptor molecule for its amide nitrogen - and just such a result was obtained. Deamination during the starvation period would also explain why the $\mathrm{pH}$ of the culture increased during this period. Carbon dioxide stripping from the medium also contributed to bringing about this $\mathrm{pH}$ change. Conversely the $\mathrm{pH}$ drop that was observed following each methanol addition was due to a combination of ammonia assimilation and carbon dioxide production.

This work has a number of wide-ranging implications for fermentation practice. For example, most continuous culture apparatus is designed in such a way that drops of media enter the culture vessels at a frequency that depends upon the imposed growth rate. At low growth rates the intervals between additions become extended and, because of this, lowered yields might be expected, and indeed lowered yields at low growth rates have often been observed, and have been explained in terms of endogenous respiration (Herbert, 1958). The lowered yields obtained with Pseudomonas methylotropha growing at low dilution rates in a carbon-limited culture where methanol was added dropwise, could, however, be explained in terms of the discontinuous growth phenomenon described above. Of interest is whether glucose-grown cultures behave similarly to methanol-grown P. methylotropha, or whether this phenomenon is peculiar to methanol-grown organisms.

A further observation that could be of considerable practical importance concerns the growth of organisms in very large continuous fermenters. In such fermenters mixing is unlikely to be perfect, as is assumed in most theoretical treatments of continuous culture kinetics, and it is quite likely that any individual organism will in fact be growing discontinuously. If this is so then the results described in the present paper are of obvious relevance.

The results described above challenge established theories of how bacteria grow in continuous cultivation devices. If organisms growing in the apparatus that is normally used by microbiologists are in fact growing discontinuously, with bursts of growth happening with a regularity that depends on the imposed dilution rate, then the explanations offered by many authors for variations in different phenomena with 'growth rate' must be reconsidered. 


\section{REFERENCES}

Brown, C. M., MacDonald-Brown, D. And MeErs. J. L. (I973). Inorganic nitrogen assimilation by micro-organisms. Advances in Microbial Physiology (in the Press).

Dawson, P. S. S. (1965). Continuous phased growth in a modified chemostat. Canadian Journal of Microbiology II, 893-905.

Dawson, P. S. S. (1971). A pilot plant apparatus for continuous phased culture - some observations on oxygen usage by Candida utilis during the cell cycle. Biotechnology and Bioengineering 8, 877-892.

DeGN, H. \& HARRISON, D. E. F. (I969). Theory of oscillations of respiration rate in continuous culture of Klebsiella aerogenes. Journal of Theoretical Biology 22, 238-248.

Gilley, J. W. \& BungaY, H. R. (1967). Oscillatory growth rate responses of $S$. cerevisiae in continuous culture. Biotechnology and Bioengineering 9, 617-622.

GoodwIN, B. C. (1969). Synchronisation of Escherichia coli in a chemostat by periodic phosphate feeding. European Journal of Biochemistry 10, $5 \mathrm{I} \mathrm{I}-5 \mathrm{I} 4$.

HAnsche, P. E. (1969). A theoretical basis for the entrainment of chemostat populations. Journal of Theoretical Biology 24, 335-350.

Harrison, D. E. F. \& PirT, S. J. (1967). The influence of dissolved oxygen concentration on the respiration and glucose metabolism of Klebsiella aerogenes during growth. Journal of General Microbiology 46, I93-2II.

Herbert, D. (1958). Some principles of continuous culture. In Recent Progress in Microbiology, p. 38I. VIIth International Congress for Microbiology.

KogA \& Humphrey (1967). Study of the dynamic behaviour of the chemostat system. Biotechnology and Bioengineering 9, 375-387.

Maclennan, D. G., Ousby, J. C., Vasey, R. B. \& Cotton, N. T. (1971). The influence of dissolved oxygen on Pseudomonas AMI grown on methanol in continuous culture. Journal of General Microbiology 69, 395-404.

MAteles, R. I., RYu, D. Y. \& YASADA, T. (1965). Measurement of unsteady steady state growth of microorganisms. Nature, London 208, 263-265.

MEERS, J. L. (1971). Effect of dilution rate on the outcome of chemostat mixed culture experiments. Journal of General Microbiology 67, 359-36r.

MeErs, J. L. (1973). Growth of bacteria in mixed culture. In Critical Reviews in Microbiology 2, 139-183.

MeErs, J. L. \& Brooks, J. D. (I973). The effects of discontinuous methanol addition on the growth of a methanol-limited culture of Pseudomonas. Journal of General Microbiology. (ln the Press.)

MONOD, J. (1942). Recherches sur la croissance des cultures bactériennes. Paris: Herman et cie.

Nagai, S., Nishizawa, Y., Endo, I. \& Aiba, S. (I968). Response of a chemostatic culture of Azotobacter vinelandii to a delta type pulse in glucose. Journal of General and Applied Microbiology I4, I 2 I-134.

ReGan, D. L., Roper, G. H. \& Moss, F. J. (197I). Response of continuous cultures to stimuli in glucose feed rate and dilution rate. Biotechnology and Bioengineering 8, 81 5-824.

Tempest, D. W., Meers, J. L. \& Brown, C. M. (1970). Synthesis of glutamate in Aerobacter aerogenes by a hitherto unknown route. Biochemical Journal II7, 405-407.

Tempest, D. W., Meers, J. L. \& Brown, C. M. (197I). Influence of environment on the content and composition of microbial free amino acid pools. Journal of General Microbiology 64, 17I-185. 